\title{
Enhanced Attention Capture by Emotional Stimuli in Mild Traumatic Brain Injury
}

\author{
Verónica Mäki-Marttunen,, ${ }^{1, *}$ Venla Kuusinen,, ${ }^{1, *}$ Maarja Brause, Jari Peräkylä, Markus Polvivaara, \\ Rodolfo dos Santos Ribeiro, Juha Öhman,, and Kaisa M. Hartikainen ${ }^{1,2}$
}

\begin{abstract}
Mild traumatic brain injury (mTBI) may be associated with compromised executive functioning and altered emotional reactivity. Despite frequent affective and cognitive symptoms in mTBI, objective evidence for brain dysfunction is often lacking. Previously we have reported compromised performance in symptomatic mTBI patients in an executive reaction time (RT) test, a computer-based RT test engaging several executive functions simultaneously. Here, we investigated the cognitive control processes in mTBI in context of threat-related stimuli. We used behavioral measures and event-related potentials (ERP) to investigate attentional capture by task-relevant and task-irrelevant emotional stimuli during a GoNoGo task requiring cognitive control. We also assessed subjective cognitive, somatic, and emotional symptoms with questionnaires. Twenty-seven subjects with previous mTBI and 17 controls with previous ankle injury participated in the study over 9 months post-injury. Electroencephalogram (EEG) was recorded while patients performed a modified executive RT-test. N2-P3 ERP component was used as a general measure of allocated attentional and executive processing resources. Although at the time of the testing, the mTBI and the control groups did not differ in symptom endorsement, mTBI patients reported having had more emotional symptoms overall since the injury than controls. The overall RT-test performance levels did not differ between groups. However, when threat-related emotional stimuli were used as Gosignals, the mTBI group was faster than the control group. In comparison to neutral stimuli, threat-related stimuli were associated with increased N2-P3 amplitude in all conditions. This threat-related enhancement of the N2-P3 complex was greater in mTBI patients than in controls in response to Go signals and NoGo signals, independent of relevance. We conclude that mTBI may be associated with enhanced attentional and executive resource allocation to threat-related stimuli. Along with behavioral evidence for enhanced attention allocation to threat stimuli, increased brain responses to threat were observed in mTBI. Enhanced attention capture by threat-related emotional stimuli may reflect inefficient topdown control of bottom-up influences of emotion, and might contribute to affective symptoms in mTBI.
\end{abstract}

Key words: attention; EEG; emotion; mTBI; response inhibition

\section{Introduction}

M ILD TRAUMATIC BRAIN INJURY (MTBI) may sometimes be associated with prolonged affective symptoms such as irritability, enhanced emotional reactivity, anxiety, and depression, even though objective evidence for brain dysfunction is typically lacking. Depression is a common concomitant after TBI. ${ }^{1}$ In addition to a variety of psychosocial factors contributing to depression after MTBI, there is evidence for disrupted corticolimbic neural circuitries that are critical for mood regulation and emotionattention interaction, ${ }^{2}$ supporting that there are biological mechanisms behind depression after mTBI. ${ }^{3}$

Integrity of frontal circuits is critical for efficient executive functions needed for coordinating cognitive functions and creating goal-oriented, purposeful actions, ${ }^{4}$ as well as for controlling emotions and mood. ${ }^{5}$ Many of the symptoms frequently reported by mTBI patients such as distractibility and emotional reactivity, as well as light and noise sensitivity, suggest deficient frontal topdown executive and attentional control of cognitive, emotional, and sensory processes. Whereas most mTBI patients fully recover, some remain symptomatic with deficits in attentional and executive functions thought to be the result of disrupted integrity of frontal circuitries. ${ }^{6}$ In addition, there is imaging, electrophysiological, and pathophysiological evidence pointing to frontal dysfunction in mTBI. These include reports of reduced frontal activation, ${ }^{7}$ disrupted frontal functional connectivity, ${ }^{8}$ diminished frontal interhemispheric coordination, ${ }^{9}$ and microscopic frontal trauma-related pathology in mTBI. ${ }^{10}$

${ }^{1}$ Behavioral Neurology Research Unit, Tampere University Hospital, Tampere, Finland.

${ }^{2}$ Department of Neurosciences and Rehabilitation, Tampere University Hospital, Tampere, Finland.

*The first two authors contributed equally. 
An intact prefrontal cortex provides a dynamic filter that allows us to focus our attention on selected stimuli, while ignoring others. ${ }^{11}$ Because of the brain's limited pool of neural processing resources, selective attention is needed as a mechanism allowing the selection of which stimuli receive the processing resources at a given time. ${ }^{12}$ Voluntary top-down control of attention relying on frontal functions gears attentional resources to stimuli that are relevant to the current goals, whereas involuntary bottom-up mechanisms gear attention toward salient or biologically important stimuli, such as emotional stimuli. ${ }^{13}$ Fine and flexible balance between the bottom-up and top-down attentional control mechanisms allows for adaptive behaviors.

Emotional stimuli, especially those that are threat related, are thought to be prioritized for access to attentional resources because of their biological, evolutionary, and behavioral relevance. ${ }^{14,15}$ Deficient frontal functions may lead to stronger bottom-up influence of threat-related negative emotional information and inefficient top-down control of emotion, such as the reappraisal mechanism necessary for maintaining euthymic mood. Alterations in normal emotion-attention interaction, such as bias toward allocating attention to negative information, are typically seen in anxiety and depression. ${ }^{16}$

Applying computer-based attention tasks with emotional distractors allows objective assessment of emotion-attention interaction and its alterations in clinical populations with brain damage or a disorder that impacts limbic or attentional circuitries. By measuring emotional interference on task performance, we have previously observed enhanced attention allocation to threat-related distractors in epilepsy patients treated with deep brain stimulation (DBS) of the anterior thalamic nuclei (ANT). ${ }^{17}$ In accordance with this objectively measured alteration in emotion-attention interaction toward negativity bias encountered in depression, ANT-DBS treatment has been linked with subjective reports of depression symptoms. ${ }^{18}$

Even when irrelevant to the current goals, emotional stimuli tend to capture attentional resources leading to interference of task performance, ${ }^{14}$ along with reduction in target-related brain potentials ${ }^{19}$ and attention network activation. ${ }^{20}$ Event-related potentials (ERPs) are well suited for studying neural mechanisms and dynamics of emotion-attention interaction and possible alterations in its nature, magnitude, and time course as a result of brain injury. ${ }^{21,22}$ ERP evidence has provided valuable information about the role of orbitofrontal cortex in emotion-attention interaction, ${ }^{23}$ and supported the notion that frontal injury leads to deficits in top-down modulation by the anterior control system. ${ }^{22}$

For better understanding of cognitive and affective symptoms associated with mTBI and their mechanisms, it is essential to assess possible alterations in emotion-attention and emotion-executive function interactions and how they are reflected in behavior and brain physiology. Although there are some studies on the impact of mTBI on affective, attentional, and executive functions, ${ }^{21,24}$ few studies have investigated alterations in emotion-attention and emotion-executive function interactions, let alone their mechanisms. In the current study, we investigated whether attention allocation to emotional stimuli and emotion-executive function interaction are altered in patients with a history of mTBI in comparison with a demographically controlled non-head trauma group with a history of ankle injury, recruited from the same emergency room.

We investigated cognitive control processes in MTBI in the context of threat-related stimuli. We used behavioral measures and ERPs to investigate attention capture by task-relevant and task- irrelevant emotional stimuli during a task requiring cognitive control. Attention to task-relevant stimuli evokes a positive parietal ERP waveform called P3, preceded ${ }^{25}$ by a negative deflection called N2. N2-P3 components are generally implicated in attentional orientation with greater N2-P3 peak-to-peak amplitude in response to stimuli that capture more attention. Anterior frontocentral N2 amplitude has been reported to increase as the demand for cognitive control increases, ${ }^{26}$ whereas parietal P3 amplitude has been suggested to reflect increased attentional resources to motivationally significant stimuli. ${ }^{27}$ In order to investigate emotionattention and emotion-cognitive control interaction, and how these interactions might be altered after mTBI, we used N2-P3 peak-topeak amplitude as a general measure of allocated processing resources. More specifically, central N2 peak was used as an index of cognitive control, and parietal P3 as an index of allocated attentional resources. With vulnerability to depression and frequent affective symptoms in mTBI, we hypothesized that weaker frontal top-down control of emotional signals in mTBI would enhance attention allocation to threat-related emotional stimuli evidenced by enhanced effect of emotional stimuli on behavior and ERPs.

\section{Methods}

\section{Subjects}

Patients admitted to the Tampere University Hospital emergency room between January 2010 to May 2012 for mTBI $(n=27)$ or ankle injury (controls, $n=17$ ) were recruited. The groups were matched in age, sex, and level of education. (mTBI: 12 females, 15 males, mean age 41 years, mean education 14.5 years; controls: 11 females, 6 males, mean age 40 years, mean education 15.8 years). The inclusion criteria for mTBI used in this study were those of the World Health Organization Center Force on mTBI. ${ }^{28}$ These diagnostic criteria for MTBI include: 1) biomechanical force applied to the head resulting in loss or alteration of consciousness, confusion, and/or post-traumatic amnesia (PTA); 2) loss of consciousness if present for <30 min, Glasgow Coma Scale (GCS) score 13-15 after $30 \mathrm{~min}$ following injury; and 3) if PTA, duration $<24 \mathrm{~h}$. Exclusion criteria included previous psychiatric or neurological disorder or substance abuse. All of the patients provided a written informed consent according to the declaration of Helsinki. The study was approved by the Ethical Committee of the Tampere University Hospital.

The average time from the injury to the research visit including the electroencephalogram (EEG) recording, computer-based emotional NoGo task, and the questionnaires, was 20.4 months (range 9-37 months) post-injury.

\section{Questionnaries}

All of the participants filled in questionnaires including demographic information, Behavior Rating Inventory of Executive Function Adult version (BRIEF-A), Rivermead Post Concussion Symptoms Questionnaire, and Beck's Depression Inventory (BDI). The BRIEF-A assesses subjective view of everyday behaviors associated with executive functioning. The Rivermead Post Concussion Symptoms Questionnaire (RPCSQ) ${ }^{29}$ lists the symptoms most commonly associated with brain injuries; for example, headache, nausea, dizziness and depression. $\mathrm{BDI}^{30}$ is a 21 question inventory developed for assessment of possible depressive symptoms and their severity.

\section{Modified executive reaction time $(R T)$ test}

A computer-based Go-NoGo visual discrimination paradigm used in this study was modified from an executive RT test that has been previously shown to detect subtle executive impairment after 
mild brain injury, ${ }^{24}$ as well as alterations in emotion-attention interaction caused by deep brain stimulation in epilepsy patients. ${ }^{17}$ The executive RT test requires multiple executive functions to be engaged simultaneously, such as inhibition, shifting, emotional control, and working memory. In the modified executive RT test used in this study, emotional stimuli served as task-irrelevant distractors as well as task-relevant Go or NoGo signals (Fig. 1). This allows for investigating the capture of attentional resources by emotional stimuli when they are competing with the task demands (distractors) as well as when they are central to the task demands (signals).

We used black line drawings forming an image of a spider (emotional distractor/signal) as threat-related emotional stimuli, and emotionally neutral control stimuli that were constructed from identical line components rearranged in a different configuration forming an image of a flower (neutral distractor/signal). Line drawings were used in order to control for other visual features such as color, brightness, contrast, spatial frequency, and complexity. These stimuli have been previously shown to evoke emotionrelated behavioral and electrophysiological responses both in healthy subjects ${ }^{34}$ and in patient populations. ${ }^{31}$

The subjects were seated in front of a computer screen at a distance of $1 \mathrm{~m}$. A computer program (Presentation, Neurobehavioral Systems, Inc.) presented the paradigm and collected the response data. The paradigm consisted of upright- and downwardfacing triangles presented for $150 \mathrm{msec}$ in a random order. There were two different kinds of blocks: one where emotional stimuli were relevant and one where they were irrelevant. In half of the blocks where emotion stimuli were relevant, the spider signified a Go signal and the flower a NoGo signal. In half of the blocks this rule was reversed. When emotional stimuli were task-irrelevant distractors, the color (red or green) on the background of the spider or flower served as the Go or NoGo signal. Subjects had to flexibly shift between these four different rules for responding, keep the response rule in working memory, and inhibit responding according to the previous rule. After a Go signal, the subjects had to press a button with their index finger if the triangle had been facing downwards and with their middle finger if the triangle had been facing upwards. Subjects had to hold the orientation of the triangle in their working memory, as the triangle had already disappeared from the screen when a Go signal appeared. The subjects were instructed to answer as fast and accurately as possible. One block consisted of 64 trials, and the subjects completed a total of 16 blocks: four of each different block type. The block type and, therefore, the rule for answering, changed between every block. Response hands were counterbalanced.

\section{EEG recording and processing}

EEG was recorded with 64 channel actiCAP Ag/AgCl electrodes (Gilching, Germany). EEG was digitized with a $512 \mathrm{~Hz}$ sampling rate. The impedance of the electrodes was below $5 \mathrm{k} \Omega$. The ERP analysis was conducted with Brain Vision Analyzer 2 (Brain Products, Gilching, Germany). The EEG data were referenced offline to linked mastoids (TP9 and TP10) and band-pass filtered (0.5$30 \mathrm{~Hz} ; 24 \mathrm{~dB} /$ octave). Eye movements were corrected based on a semiautomatic independent component analysis. Epochs with amplitudes $> \pm 100 \mu \mathrm{V}$ were rejected. Single trials from $200 \mathrm{msec}$ pre- to $1800 \mathrm{msec}$ post-stimulus (triangle) presentation were averaged for each condition.

\section{Behavioral analysis}

Mean values of RT and number of errors were measured for the different conditions and for each subject. There were three different error types: incorrect button presses, commission errors, and missed responses. An incorrect response indicated an incorrect button press to the orientation of the triangle during a Go trial. Incorrect error is thought to reflect lapse in working memory or attention performance. A commission error indicated a failure in withholding from responding on a NoGo trial. A commission error is thought to reflect a failure in response inhibition. A miss indicated failure to respond during a Go trial within a given time. Misses reflected inattention and/or inability to initiate a response within the allowed time.

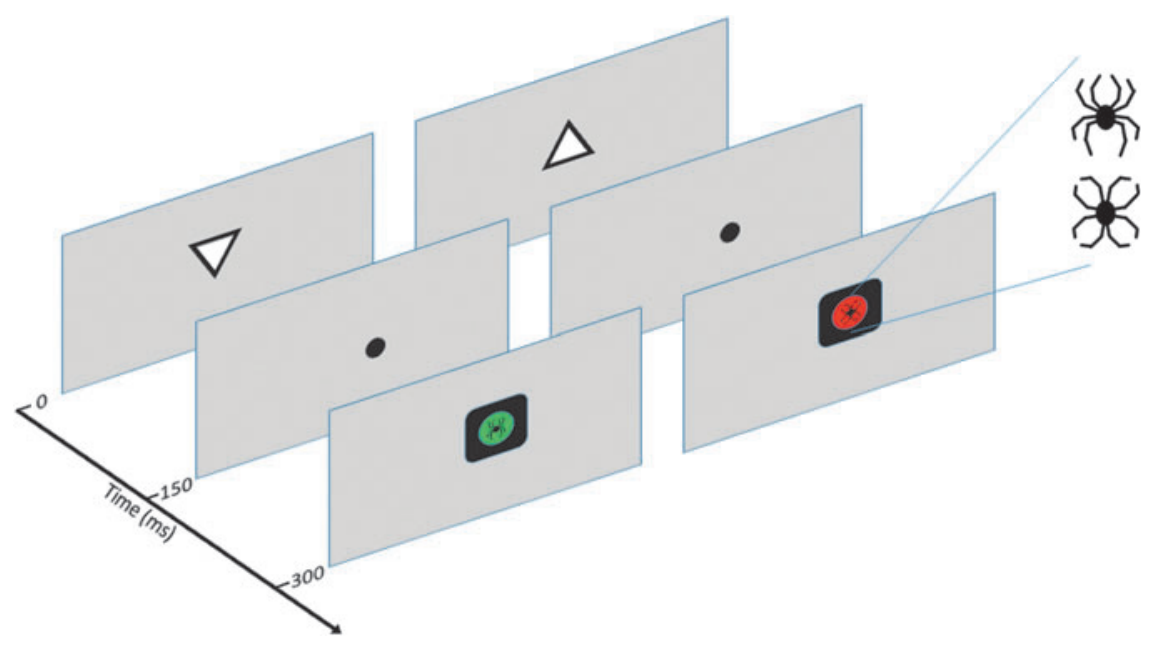

FIG. 1. Schematic presentation of the modified executive reaction time (RT) test. Example of two trials. Subjects were instructed to respond to the orientation of a triangle with a button press if a Go signal was presented after the triangle, and to withhold from responding if a NoGo signal was presented. There were four different block types with identical sets of trials presented in random order, but with different stimuli (spider, flower, green, red) serving as Go and NoGo signals. In the emotion relevant condition, either a spider was a Go-signal and a flower was a NoGo signal or vice versa. In the emotionally irrelevant condition, one of the colors (red or green) was a Go signal and the other a NoGo signal, with emotional stimuli serving as task-irrelevant distractors. Therefore, with identical stimuli presented in different block types with the only difference being stimulus relevance to the task, mere physical properties of the stimuli do not explain different effects in emotionally relevant and irrelevant conditions. Similarly, with emotional (spider) and emotionally neutral stimuli (flower) constructed from the same line components, emotional effects observed in this study cannot be explained by any lower level visual attributes. Color image is available online at www.liebertpub.com/neu 


\section{ERP analysis}

ERPs were averaged according to valence of the emotional stimuli (threat [NEG] or neutral [NEU]), relevance of the emotional stimuli for the task (relevant $[\mathrm{R}]$ or not relevant $[\mathrm{NR}]$ ) and the response signal (Go or NoGo). With visual inspection of the grand average waveforms, we identified $\mathrm{N} 2$ and $\mathrm{P} 3$ peaks (see Fig. 2). The emotion relevant condition led to longer reaction times and delayed $\mathrm{N} 2$ and P3 ERP peaks in comparison with the emotion irrelevant condition. This was probably because the line drawing discrimination required in the emotion relevant condition was a harder task than the color discrimination required in the emotion irrelevant condition. Therefore, slightly different time windows for ERP peak detection were used for the emotion relevant and irrelevant conditions. The time windows were expressed from the onset of the trial; that is, from the onset of the triangle presentation. The onset of the Go-NoGo signal/Emotional stimuli was at $300 \mathrm{msec}$ from the trial onset. The time windows used for peak detection were 550 $700 \mathrm{msec}(\mathrm{R}), 500-650 \mathrm{msec}(\mathrm{NR})$ for N2 and 700-800 msec (R), 650-750 (NR) for P3. We used N2-P3 peak-to-peak amplitude as a general index of attentional and executive processing resource allocation. Peak-to-peak amplitude measurement allows for subtracting the effect of slow positive or negative ERP deflections caused by other cognitive processes being concurrent with N2 and P3 peaks, thus allowing for a better estimate of overall resource allocation than a single peak measurement. We further used central N2 and parietal P3 peak amplitudes as indexes of cognitive control and attentional resources, correspondingly. The central region was chosen for investigating the anterior frontocentral N2, because the $\mathrm{N} 2$ peak amplitude maxima was centrally located.

We further explored the time course of the ERPs by dividing the interval between 550 and $850 \mathrm{msec}$ into $50 \mathrm{msec}$ time windows. The mean amplitude in each time window was extracted, and these data were used for statistical analysis. Identification of peaks and computation of mean amplitudes were performed in MATLAB 12 (Math Works Inc., Natick, MA).

\section{Statistical analysis}

SPSS (SPSS Inc., Chicago, IL) was used for statistical analysis. Normal distribution of the variables was tested statistically with the Kolmogorov-Smirnov test. Repeated measures analysis of variance (RM-ANOVA) was performed on RTs, and different error types with emotion (threat, neutral) and task relevance of the symbolic stimuli (relevant, irrelevant) as within-subject factors and group as the between-subject factor (control, mTBI).

The average scores from questionnaires for the ankle and mTBI groups were compared with the Mann-Whitney $U$ test. For RPCSQ scores, the total symptom score and separate scores for different kinds of symptoms were compared with three different categories: somatic, emotional, and cognitive symptoms.

N2-P3 peak-to-peak amplitude was used as a general measure of resource allocation. For statistical assessment of the ERPs, RMANOVA was performed to test for group differences in N2-P3 amplitude separately for the Go and NoGo conditions with emotion (threat, neutral), task relevance of the emotional stimuli (relevant,
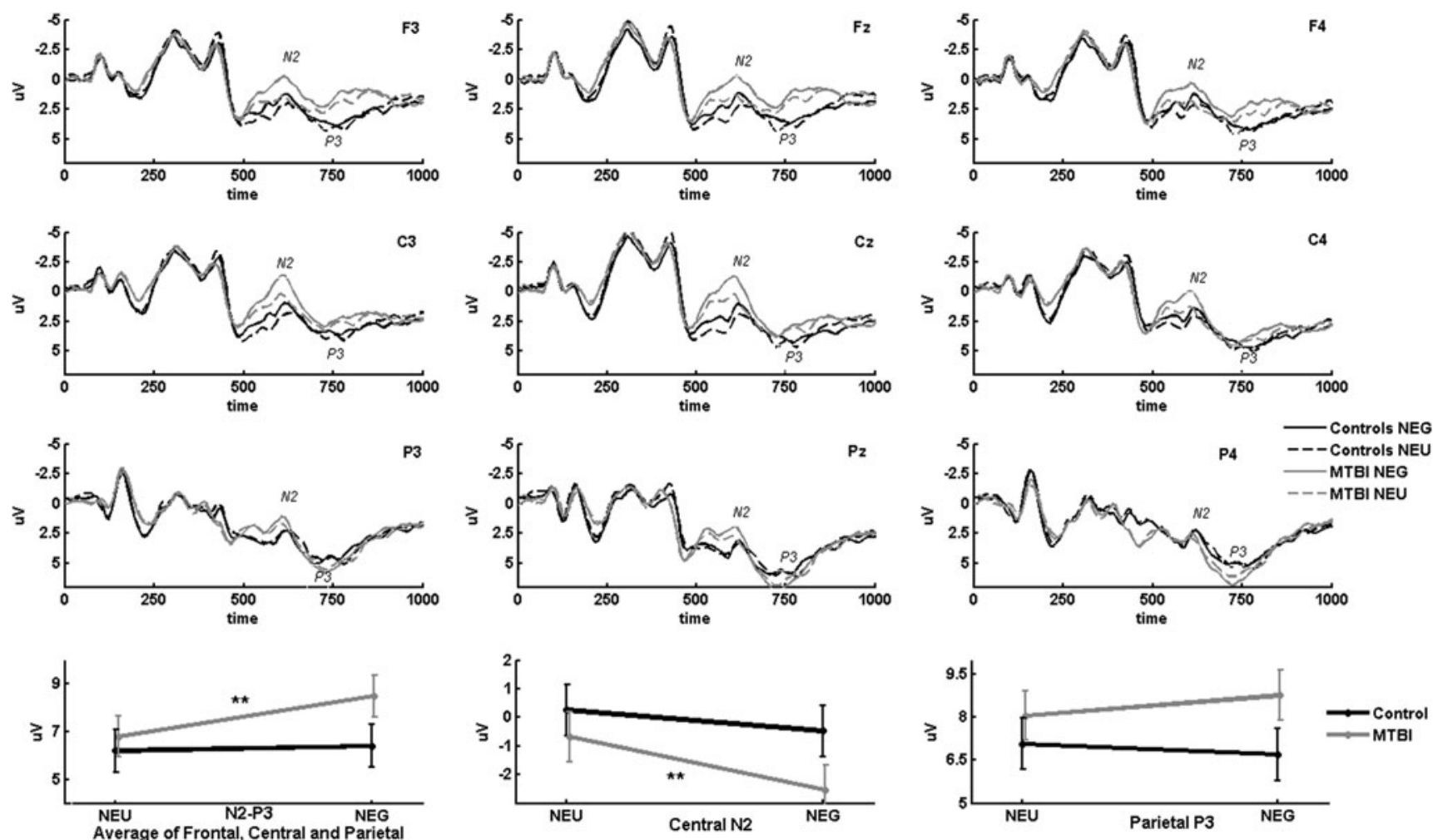

FIG. 2. Greater attention capture by task-relevant emotional stimuli in mild traumatic brain injury (mTBI). Threat-related Go signals evoked greater N2-P3 amplitude than neutral signals in the mTBI group than in the control group. Parietal P3 showed a trend, and central N2 showed a significant threat-related enhancement in the mTBI group. Grand average event-related potentials (ERPs) in response to emotionally relevant Go trials are illustrated from nine electrodes that were used to form frontal (F3, Fz, F4), central $(\mathrm{C} 3$, $\mathrm{Cz}, \mathrm{C} 4)$, and parietal (P3, Pz, P4) regions of interest for statistical analysis. The bottom plots from left to right illustrate 1) average N2P3 peak-to-peak amplitudes across all regions of interest (frontal, central, parietal); 2) central N2 peak; and 3) parietal P3 peak amplitudes for mTBI and control groups in response to emotional (NEG) and emotionally neutral (NEU) control stimuli. Error bars indicate standard error. $* * p<0.001$. 
irrelevant), and EEG brain region (frontal, central, parietal) as within-subject factors, and group (control, mTBI) as the betweensubject factor. To reduce the number of statistical comparisons and to improve signal-to-noise ratio, we clustered electrodes over regions of interest: frontal (F3, Fz, F4), central $(\mathrm{C} 3, \mathrm{C} 4, \mathrm{Cz})$, and parietal $(\mathrm{P} 3, \mathrm{Pz}, \mathrm{P} 4)$ areas. Because parietal P3 is especially implicated in attentional allocation, ${ }^{25}$ and frontal $\mathrm{N} 2$ is implicated in control processes, ${ }^{32}$ we conducted a separate RM-ANOVA analysis for P3 peak amplitude in the parietal region and N2 peak amplitude in the frontal region with emotion (threat, neutral) and task relevance of the symbolic stimuli (relevant, irrelevant) as withinsubject factors, and group (control, mTBI) as the between-subject factor.

\section{Results \\ Questionnaires}

There was a significant difference between the groups in the RPCSQ overall emotional symptom score, with the mTBI group reporting more emotional symptoms $(1.81 \pm 3.16)$ than the control group $(0.41 \pm 1.28, p<0.05)$. The symptoms categorized as emotional were depressed/fearful feelings, frustration/impatience, irritability/getting angry easily, and restlessness. There were no other significant differences between groups in any of the other questionnaire scores including the BDI score, BRIEF-A total score, and BRIEF-A subscores for different executive functions.

\section{Electrophysiological results}

The irrelevant condition was associated with better performance than the relevant condition, including faster RTs $(F=119, p=0.05$, effect size: 0.81$)$. The groups performed the task at comparable levels in general, with no difference in RTs between the groups. However, mTBI patients were faster than controls in the emotion relevant condition (post-hoc $t$ test in the relevant condition, mTBI vs. controls: $T=2.13, p=0.039$, effect size $=0.58)$. Threat-related stimuli were associated with faster RTs than neutral ones $(T=4.92$, $p<0.001$, effect size $=0.09$ ). Speeding of RTs was significant only when emotional stimuli were task relevant; that is, threat-related Go signals (post-hoc $t$ test in the relevant condition, threat vs neutral: $T=5.23, p<0.001$, effect size $=0.17$ ), and only in the mTBI group (post-hoc $t$ test in mTBI in the relevant condition, threat vs neutral: $T=6.59, p<0.001$, effect size $=0.24$ ). The groups had also comparable performance levels with regard to accuracy, with no main effect of group for error analysis. There was an interaction effect of emotion by relevance by group $(F=4.33$, $p=0.043$ ) for commission errors, with the mTBI group making more commission errors in the context of threat-related distractors compared to controls (post-hoc $t$ test, threat, mTBI vs. controls: $T=2.089, p=0.044$, effect size $=1.09$ ).

Threat-related stimuli evoked greater N2-P3 amplitude in both Go and NoGo trials, suggesting enhanced allocation of processing resources caused by emotional stimuli in general (Go: $F=19.2$, $p<0.001$, effect size $=0.18 ;$ NoGo: $F=15.8, p<0.001$, effect size $=0.2$ ). Enhanced resource allocation to emotional stimuli was seen only in the mTBI group with task-relevant emotional Go signals evoking greater N2-P3 amplitude than neutral ones (Fig. 2) (interaction effect emotion by group: $p=0.013$; post-hoc $t$ test in mTBI, threat vs. neutral: $T=5.53, p<0.001$, effect size $=0.42$ ). In controls, significant emotional enhancement of N2-P3 peak-topeak amplitude was not observed. Emotional enhancement of N2P3 amplitudes were further investigated by separate analysis of central N2 and parietal P3, indexing cognitive control and atten- tional resources respectively. In contrast to controls, N2 peak amplitude was significantly enhanced by emotional Go signals in the mTBI group (Fig. 2) (N2, interaction effect emotion by group: $F=8.13, p=0.007$; post-hoc $t$ test in mTBI, threat vs. neutral: $T=7.3, p<0.001$, effect size $=0.45$ ). The emotional enhancement of parietal P3 peak was approaching significance (interaction effect emotion by group: $F=4.32, p=0.044$; post-hoc $t$ test in mTBI, threat vs. neutral: $T=2.00, p=0.056$, effect size $=0.14$ ).

The time window analysis showed that in the Go situation, and when emotional stimuli (spider or flower) were relevant to the task, there was a significantly greater negativity in response to threatrelated than to neutral stimuli from 550 to $600 \mathrm{msec}$ in both groups. Importantly, during the 600-650 msec interval, this difference was still present in the mTBI group but not in controls. The greater negativity lasted longer in the frontal plane in the mTBI group than in controls.

NoGo trials engaging inhibitory cognitive control processes evoked greater N2-P3 amplitude in the context of threat-related stimuli. This threat-related enhancement of NoGo N2-P3 average amplitude derived from the nine electrodes in the fronto-centroparietal region was significant only in the mTBI group (Fig. 3) (interaction effect emotion by group: $F=6.29, p=0.016$; post-hoc $t$ test in mTBI, threat vs. neutral: $T=4.59, p<0.001$, effect size $=$ 0.32). Separate analysis on the contribution of the central N2 to this emotion-related N2-P3 enhancement showed that central N2 for NoGo trials was significantly enhanced by a threat-related context in both the mTBI and control groups, with a trend toward a greater effect in the mTBI group (emotion main effect: $F=30.54$, $p<0.001$; interaction effect emotion by group: $F=3.92, p=0.054$; post-hoc $t$ test in mTBI, threat vs. neutral: $T=5.36, p<0.001$, effect size $=0.29$; post-hoc $t$ test in controls, threat vs. neutral: $t=2.66$, $p=0.016$, effect size $=0.16$ ). There were no significant group or emotion effects for parietal P3 in NoGo trials.

\section{Discussion}

This study provides novel evidence for altered emotion-attention and emotion-executive function interaction in patients with a history of mTBI. mTBI group allocated more attentional resources to emotional stimuli, as was evidenced by the greater influence of emotional stimuli on both behavior and brain physiology. We reported enhanced attention allocation to threat-related stimuli evidenced by increased N2-P3 amplitudes in the mTBI group. Furthermore, in the mTBI group, enhanced attention allocation to threat-related stimuli led to improved performance when the emotional cue was relevant to the task, and impaired performance when the emotional stimuli was irrelevant to the task.

Robust brain mechanisms within the limbic circuitry support survival by allowing for automatic attention allocation to biologically threatening stimuli such as spiders. Bottom-up influence of emotionally threatening stimuli is controlled by top-down mechanisms suppressing undue emotional reactivity. In the current study, we used line drawings of spiders to study threat-related attention allocation. In order to control for low-level visual features, line drawings of spiders were used as emotional stimuli, whereas the emotionally neutral control stimuli were constructed from identical visual components, but in a different configuration. Identical low-level visual attributes are unobtainable with natural scenes or photographs. Emotional stimuli depending upon low visualspatial frequency information have been shown to readily activate the amygdala. ${ }^{33}$ Furthermore, similar line drawings of spiders have been shown to have robust influence on attention networks and 

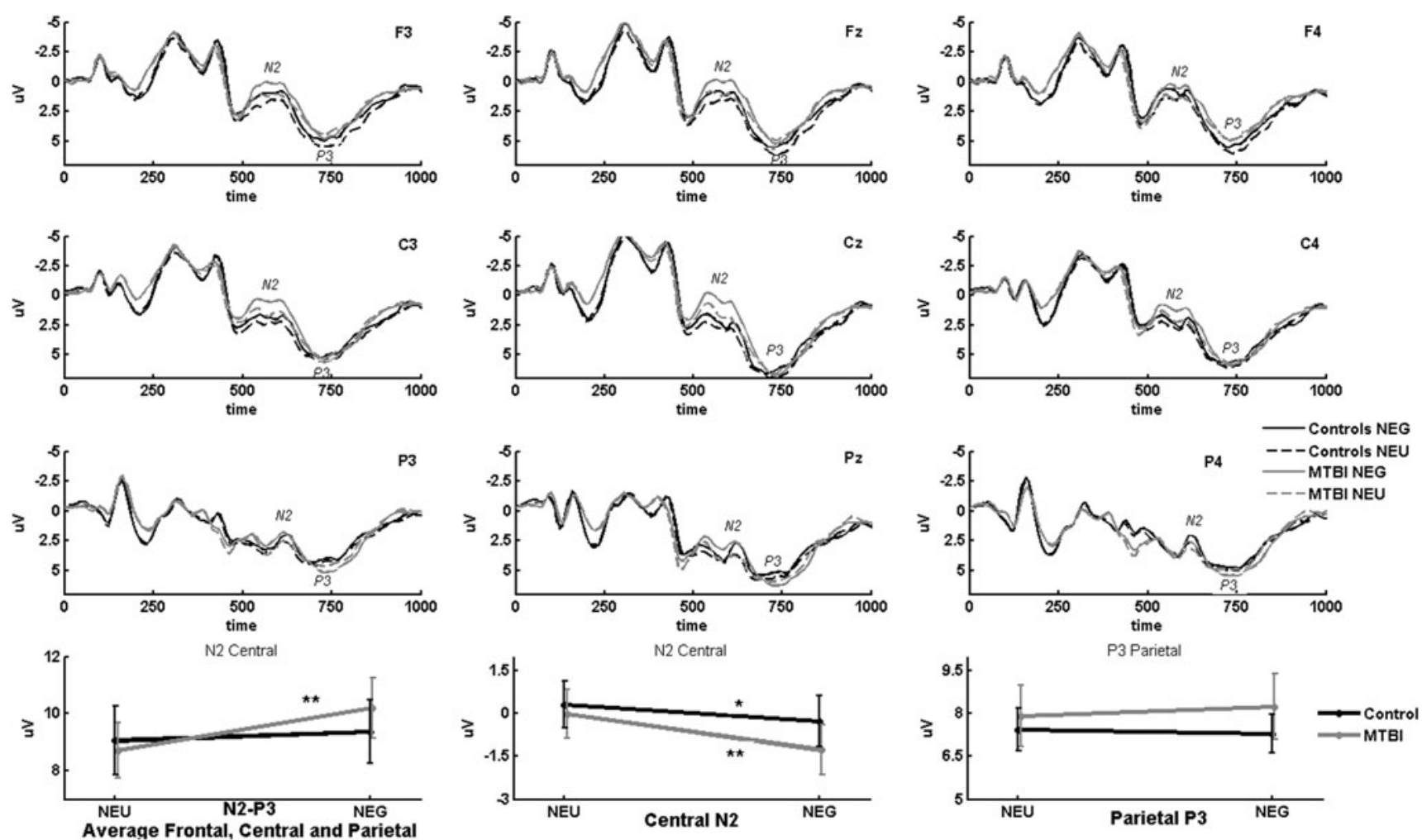

FIG. 3. Altered emotion-cognitive control interaction in mild traumatic brain injury (mTBI). Threat-related stimuli were associated with greater N2-P3 amplitude in response to NoGo trials in the mTBI group than were neutral stimuli. Central N2 enhancement in response to emotional stimuli was observed both in the mTBI group and in controls, with a tendency toward greater effect in mTBI. Grand average event-related potentials (ERPs) evoked by all NoGo trials are illustrated in the left, midline, and right frontal (F3, Fz, F4), central $(\mathrm{C} 3, \mathrm{Cz}, \mathrm{C} 4)$, and parietal $(\mathrm{P} 3, \mathrm{Pz}, \mathrm{P} 4)$ electrodes that were used to obtain amplitude measurements for the three regions of interest. Bottom plots from left to right illustrate 1) average N2-P3 peak-to-peak amplitudes across fronto-centro-parietal region, 2) central N2 peak, and 3) parietal P3 peak amplitudes for mTBI and control groups in response to emotional (NEG) and emotionally neutral (NEU) control stimuli in all NoGo trials. Error bars indicate standard error. ${ }^{*} p<0.05 ; * *<<0.001$.

behavior. ${ }^{17,31,34}$ Therefore, the spider stimuli used in this study presumably activated automatic threat-related processes in the limbic circuitries with prioritized access to attention networks. Despite the challenges of controlling for other visual attributes when using more natural emotional and social stimuli, future studies with such stimuli will provide important further insight into emotion-attention interaction and its possible alterations in $\mathrm{mTBI}$ patients.

mTBI patients allocated more attentional resources to emotional stimuli than did control subjects, both when emotional stimuli were relevant and when they were irrelevant to the task. Attention allocation to an emotionally relevant condition seemed to be enhanced in mTBI patients in contrast to control subjects, with enhanced response speed and increased ERPs in response to emotionally relevant Go trials. When attention allocation was enhanced to emotional stimuli that were relevant to the task, performance improved. On the contrary, when emotional stimuli were irrelevant to the task and they competed for the attentional resources with task-relevant stimuli, task performance suffered. In NoGo trials, emotional stimuli independent of relevance captured attentional resources more than neutral stimuli, and to a greater extent in the mTBI group than in controls, as evidenced by an enhanced N2-P3 NoGo response. Attention allocation to threatrelated stimuli was disruptive to behavior when emotional stimuli were distractors. mTBI patients made more commission errors; that is, errors in which response inhibition failed in the context of emotional distractors.

The phenomenon called "negativity bias" suggests that allocation of attentional resources is biased toward negative stimuli in general. ${ }^{35}$ This is in accordance with our findings with both groups being faster with relevant emotional stimuli, and experiencing greater negativity in response to threat-related stimuli than to neutral stimuli in the ERPs between 550 and $600 \mathrm{msec}$ in a Go situation, indicating that threat recruits more attentional resources than do neutral distractors in general. In our study, it seemed as if mTBI led to even stronger prioritization and prolonged processing of emotionally negative, threat-related stimuli. In the mTBI group only, the enhanced negativity evoked by emotional stimuli was still present between 600 and $650 \mathrm{msec}$, reflecting prolonged attention allocation to emotional stimuli or delayed disengagement of attention away from emotional stimuli back to the task.

The prefrontal cortex is thought to be susceptible to brain injury. Inefficiently functioning frontal circuitries in mTBI might reduce frontal filtering of or frontal top-down control of emotional signals, leading to greater attention allocation to and impact of emotional stimuli. Emotional stimuli call for the executive processing resources required for controlling emotional reactivity and adjusting behavior appropriately. Even though affective and higher cognitive control functions depend upon distinct neural circuitries, these circuitries and their functions are intricately interconnected, ${ }^{36}$ with 
bidirectional interactions. The dual competition framework suggests that emotional content creates competition between executive functions and the perceptional resources, resulting in either impaired or improved task performance. ${ }^{37}$ Emotional stimuli can either enhance or impair task execution depending upon several emotion-, task-, and subject-related factors. ${ }^{14,19,31,34,38}$ Therefore, processing resources needed for performing executive functions required by the task may be compromised by unexpected engagement of executive functions by emotional stimuli. We have previously shown that in healthy subjects, threat-related stimuli lead to compromised inhibitory executive control functions, as evidenced by increased commission errors in a context of threat-related distractors. ${ }^{34}$ In the current study, although the overall performance of the mTBI patients was comparable with that of the control group, it may be that the task exhausted the executive processing resources in the mTBI group, leaving fewer available resources for top-down control of emotional stimuli, thus allowing for greater impact of emotional stimuli on behavior and brain responses.

Attentional control relying on the prefrontal cortex and the cingulum allows diminished attention allocation to emotional stimuli. ${ }^{39}$ Decreased functional connectivity in brain regions controlling the emotion regulation; that is, between the rostral anterior cingulate cortex (rACC) and the amygdala, has been reported in TBI. ${ }^{40} \mathrm{En}-$ hanced bottom-up influence of threat-related stimuli caused by deficient top-down control, especially when control resources are exhausted, might be one of the mechanisms of emotional symptoms after mTBI. Evidence for high trait anxiety causing increased attention allocation to negative or threat-related stimuli, and an inability to actively recruit prefrontal control mechanisms to inhibit this effect have been reported. ${ }^{41}$ Even though in this study, mTBI patients did not significantly differ in depression scores from controls, high depression rates, even without a previous history of depression $^{42}$ and regardless of the severity of the injury, seem to be a notorious consequence of TBI. ${ }^{43}$ In our study, the mTBI group reported having more emotional symptoms including irritability, depression, lack of patience, and restlessness in the RPCSQ after the injury than the control group. This difference in emotional symptom scores was seen when all emotional symptoms, including those that were not currently present, were counted in the score.

The N2-P3 complex of ERPs is thought to reflect response inhibition in a Go-NoGo task. ${ }^{44}$ We detected a greater N2-P3 amplitude in response to threat-related stimuli in a NoGo situation in the mTBI group. It has been argued that in a NoGo emotional situation, both automatic response inhibition and emotional processing are occurring simultaneously, producing a larger amplitude in P3. ${ }^{45,46}$ The larger N2-P3 amplitude in the mTBI group could, therefore, reflect stronger attentional and executive resource allocation to emotional signals.

N2-P3 amplitude has been linked with the amount of attention allocation, as indexed with subsequent viewing time to novel stimuli. $^{32}$ One of the benefits of N2-P3 peak-to-peak amplitude measurement as an index of processing resource allocation is that it allows for subtracting the effect of slow ERP deflections caused by other cognitive processes concurrent with $\mathrm{N} 2$ and $\mathrm{P} 3$ peaks. As indexed with N2-P3 amplitude, we found evidence for greater processing resources allocated by mTBI patients in the context of threat both in Go and in NoGo trials. In Go trials, this emotional enhancement was seen only when emotional stimuli were relevant to the task.

In a separate analysis of the N2 and P3 peaks, we found enhanced emotional modulation of both central N2 and parietal P3 in response to Go signal in the mTBI Group. Parietal P3 amplitude has been suggested to reflect increased attentional resources to moti- vationally significant stimuli, ${ }^{25,27}$ whereas frontocentral $\mathrm{N} 2$ has been linked to many different cognitive processes, with cognitive control encompassing majority of them. ${ }^{26}$ Future studies, with careful analysis of the different cognitive control processes potentially temporarily overlapping with and contributing to the emotional modulation of frontocentral $\mathrm{N} 2$, are needed for deeper understanding of the mechanisms of the altered emotion-cognitive control interaction in mTBI observed in this study. However, in the framework of $\mathrm{N} 2$ reflecting the amount of top-down control needed for the task performance, N2 enhancement caused by emotion might reflect the additional top-down control needed in the face of threat-related signals in general. Central N2 amplitude was also augmented by emotion in NoGo trials, with a trend toward enhanced effect in the MTBI group. Although we found evidence for enhanced attention allocation, we also found evidence for enhanced allocation of control resources in response to threat in $\mathrm{mTBI}$, as indexed by emotional enhancement of both parietal P3 and central N2 amplitudes. Greater allocation of control resources might be a compensatory mechanism to overcome less efficient cognitive control processes in mTBI.

In the light of the current results, mTBI patients allocated more attentional and cognitive control resources to threat. In general, the mTBI patients performed at comparable levels to controls, and even better than controls when the emotional signal was relevant to the task. However, there might be a cost to the excessive allocation of attentional and control resources to threat. Excess allocation of attentional resources to threat might contribute to the emotional symptoms, and make mTBI patients more susceptible to depression.

Although the MTBI group reported more emotion related symptoms in post-concussion questionnaire (RPCS), the groups did not differ in depression measures, overall symptoms scores, or their subjective report of everyday executive functioning as measured with BRIEF. However, both the attention performance measures and the brain's evoked responses point toward enhanced emotional reactivity in the mTBI group.

\section{Conclusion}

mTBI may be associated with enhanced allocation of attentional and executive resources to threat-related stimuli. In addition to behavioral evidence for enhanced attention allocation to threatrelated stimuli, increased brain responses to threat were observed in mTBI. Enhanced attention capture by threat-related emotional stimuli may reflect threat detection bias, and might contribute to affective symptoms in MTBI. This study highlights the need for further studies in emotion-attention interaction in MTBI. Further, our study introduces a way to objectively assess emotional reactivity as reflected in behavior and brain physiology in mTBI.

\section{Acknowledgments}

This research was supported by the Academy of Finland and the Competitive Research Fund of Pirkanmaa Hospital District.

\section{Author Disclosure Statement}

No competing financial interests exist.

\section{References}

1. Bombardier, C.H., Ehde, D.M., Stoelb, B., and Molton, I.R. (2010). The relationship of age-related factors to psychological functioning among people with disabilities. Phys. Med. Rehabil. Clin. N. Am. 21, 281-297. 
2. Mayberg, H.S., Liotti, M., Brannan, S.K., McGinnis, S., Mahurin, R.K., Jerabek, P.A., et al. (1999). Reciprocal limbic-cortical function and negative mood: converging PET findings in depression and normal sadness. Am. J. Psychiatry 156, 675-682.

3. Matthews, S.C., Strigo, I.A., Simmons, A.N., O'Connell, R.M., Reinhardt, L.E., and Moseley, S.A. (2011). A multimodal imaging study in U.S. veterans of Operations Iraqi and Enduring Freedom with and without major depression after blast-related concussion. Neuroimage 54, Suppl. 1, S69-75.

4. Funahashi, S. (2001). Neuronal mechanisms of executive control by the prefrontal cortex. Neurosci. Res. 39, 147-165.

5. Spinella, M. (2007). Measuring the executive regulation of emotion with self-rating scales in a nonclinical population. J. Gen. Psychol. 134, 101-111.

6. Lipton, M.L., Gulko, E., Zimmerman, M.E., Friedman, B.W., Kim, M., Gellella, E., et al. (2009). Diffusion-tensor imaging implicates prefrontal axonal injury in executive function impairment following very mild traumatic brain injury. Radiology $252,816-824$.

7. Bonne, O., Gilboa, A., Louzoun, Y., Kempf-Sherf, O., Katz, M., Fishman, Y., et al. (2003). Cerebral blood flow in chronic symptomatic mild traumatic brain injury. Psychiatry Res. 124, 141-152.

8. Vakhtin, A.A., Calhoun, V.D., Jung, R.E., Prestopnik, J.L., Taylor, P.A., and Ford, C.C. (2013). Changes in intrinsic functional brain networks following blast-induced mild traumatic brain injury. Brain Inj. 27, 1304-1310.

9. Sponheim, S.R., McGuire, K.A., Kang, S.S., Davenport, N.D., Aviyente, S., Bernat, E.M., and Lim, K. O. (2011). Evidence of disrupted functional connectivity in the brain after combat-related blast injury. Neuroimage 54, Suppl. 1, S21-29.

10. Bigler, E.D. (2004). Neuropsychological results and neuropathological findings at autopsy in a case of mild traumatic brain injury. J. Int. Neuropsychol. Soc. 10, 794-806.

11. Shimamura, A.P. (2000). Toward a cognitive neuroscience of metacognition. Conscious. Cogn. 9, 313-323.

12. Desimone, R., and Duncan, J. (1995). Neural mechanisms of selective visual attention. Annu. Rev. Neurosci. 18, 193-222.

13. Corbetta, M., and Shulman, G.L. (2002). Control of goal-directed and stimulus-driven attention in the brain. Nat. Rev. Neurosci. 3, 201-215.

14. Hartikainen, K.M., Ogawa, K.H., and Knight, R.T. (2000). Transient interference of right hemispheric function due to automatic emotional processing. Neuropsychologia 38, 1576-1580.

15. Ohman, A., Flykt, A., and Esteves, F. (2001). Emotion drives attention: detecting the snake in the grass. J. Exp. Psychol. Gen. 130, 466-478.

16. Matthews, G., and Wells, A. (2000). Attention, automaticity, and affective disorder. Behav. Modif. 24, 69-93.

17. Hartikainen, K., Sun, L., Polvivaara, M., Brause, M., Lehtimäki, K. Haapasalo, J., et al. (2014). Immediate effects of deep brain stimulation of anterior thalamic nuclei on executive functions and emotion-attention interaction in humans. J. Clin. Exp. Neuropsychol. 36, 540-550.

18. Fisher, R., Salanova, V., Witt, T., Worth, R., Henry, T., Gross, R., et al. (2010). Electrical stimulation of the anterior nucleus of thalamus for treatment of refractory epilepsy. Epilepsia 51, 899-908.

19. Hartikainen, K.M., Ogawa, K.H., and Knight, R.T. (2010). Trees over forest: unpleasant stimuli compete for attention with global features. Neuroreport 21, 344-348

20. Mäki-Marttunen, V., Pickard, N., Solbakk, A-K., Ogawa, K.H., Knight, R.T., and Hartikainen, K.M. (2014) Low attentional engagement makes attention network activity susceptible to emotional interference. Neuroreport 25, 1038-1043.

21. Solbakk, A.K., Reinvang, I., and Nielsen, C.S. (2000). ERP indices of resource allocation difficulties in mild head injury. J. Clin. Exp. Neuropsychol. 22, 743-760.

22. Solbakk, A.K., Reinvang, I., Svebak, S., Nielsen, C.S., and Sundet, K. (2005). Attention to affective pictures in closed head injury: eventrelated brain potentials and cardiac responses. J. Clin. Exp. Neuropsychol. 27, 205-223.

23. Hartikainen, K.M., Ogawa, K.H., and Knight, R.T. (2012). Orbitofrontal cortex biases attention to emotional events. J. Clin. Exp. Neuropsychol. 34, 588-597.

24. Hartikainen, K.M., Waljas, M., Isoviita, T., Dastidar, P., Liimatainen, S., Solbakk, A. K., et al. (2010). Persistent symptoms in mild to moderate traumatic brain injury associated with executive dysfunction. J. Clin. Exp. Neuropsychol. 32, 767-774.

25. Polich, J. (2007). Updating P300: an integrative theory of P3a and P3b. Clin. Neurophysiol. 118, 2128-2148.
26. Folstein, J.R., and Van Petten, C. (2008). Influence of cognitive control and mismatch on the N2 component of the ERP: a review. Psychophysiology 45, 152-170.

27. Schupp, H.T., Stockburger, J., Codispoti, M., Junghöfer, M., Weike, A.I., and Hamm, A.O. (2007). Selective visual attention to emotion. J. Neurosci. 27, 1082-1089.

28. Carroll, L.J., Cassidy, J.D., Holm, L., Kraus, J., Coronado, V.G.; WHO Collaborating Centre Task Force on Mild Traumatic Brain Injury (2004). Methodological issues and research recommendations for mild traumatic brain injury: the WHO Collaborating Centre Task Force on Mild Traumatic Brain Injury. J. Rehabil. Med. 43, 113-125.

29. King, N.S., Crawford, S., Wenden, F.J., Moss, N.E.G., and Wade, D.T. (1995). The Rivermead Post Concussion Symptoms Questionnaire: a measure of symptoms commonly experienced after head injury and its reliability. J. Neurol. 242, 587-592.

30. Beck, A.T., Ward, C.H., Mendelson, M., Mock, J., Erbaugh, J. (1961) An inventory for measuring depression. Arch. Gen. Psych. 4:561-571.

31. Vuilleumier, P., and Schwartz, S. (2001). Beware and be aware: capture of spatial attention by fear-related stimuli in neglect. Neuroreport $12,1119-1122$.

32. Daffner, K.R., Scinto, L.F., Calvo, V., Faust, R., Mesulam, M.M., West, W.C., and Holcomb, P.J. (2000). The influence of stimulus deviance on electrophysiologic and behavioral responses to novel events. J. Cogn. Neurosci. 12, 393-406.

33. Vuilleumier, P., Armony, J.L., Driver, J., and Dolan, R.J. (2003). Distinct spatial frequency sensitivities for processing faces and emotional expressions. Nat. Neurosci. 6, 624-631.

34. Hartikainen, K.M., Siiskonen, A.R., and Ogawa, K.H. (2012). Threat interferes with response inhibition. Neuroreport 23, 447-450.

35. Ito, T.A., Larsen, J.T., Smith, N.K., and Cacioppo, J.T. (1998). Negative information weighs more heavily on the brain: the negativity bias in evaluative categorizations. J. Pers. Soc. Psychol. 75, 887-900.

36. Pessoa, L. (2010). Emergent processes in cognitive-emotional interactions. Dialogues Clin. Neurosci. 12, 433-448.

37. Pessoa, L. (2009). How do emotion and motivation direct executive control? Trends Cogn. Sci. 13, 160-166.

38. Hartikainen, K.M., Ogawa, K.H., Soltani, M., and Knight, R.T. (2007). Emotionally arousing stimuli compete for attention with left hemispace. Neuroreport 18, 1929-1933.

39. Ochsner, K.N., and Gross, J.J. (2005). The cognitive control of emotion. Trends Cogn. Sci. 9, 242-249.

40. Newsome, M.R., Scheibel, R.S., Mayer, A.R., Chu, Z.D., Wilde, E.A., Hanten, G., et al. (2013). How functional connectivity between emotion regulation structures can be disrupted: preliminary evidence from adolescents with moderate to severe traumatic brain injury. J. Int. Neuropsychol. Soc, 19, 911-924.

41. Bishop, S.J. (2008). Neural mechanisms underlying selective attention to threat. Ann. N. Y. Acad. Sci. 1129, 141-152.

42. Bombardier, C.H., Fann, J.R., Temkin, N.R., Esselman, P.C., Barber, J., and Dikmen, S.S. (2010). Rates of major depressive disorder and clinical outcomes following traumatic brain injury. JAMA, 303(19), 1938-1945.

43. Rapoport, M.J. (2012). Depression following traumatic brain injury: epidemiology, risk factors and management. CNS Drugs 26, 111-121.

44. Kaiser, S., Weiss, O., Hill, H., Markela-Lerenc, J., Kiefer, M., and Weisbrod, M. (2006). N2 event-related potential correlates of response inhibition in an auditory Go/Nogo task. Int. J. Psychophysiol. 61, 279282.

45. Albert, J., Lopez-Martin, S., Tapia, M., Montoya, D., and Carretie, L. (2012). The role of the anterior cingulate cortex in emotional response inhibition. Hum. Brain Mapp. 33, 2147-2160.

46. Zhang, W., and Lu, J. (2012). Time course of automatic emotion regulation during a facial Go/Nogo task. Biol. Psychol. 89, 444-449.

Address correspondence to: Kaisa M. Hartikainen, MD, PhD Behavioral Neurology Research Unit Tampere University Hospital PO Box 2000 33520 Tampere Finland

E-mail: kaisa.hartikainen@uta.fi 